\title{
A comparative study of the muscle strength and mass of the arm flexors and extensors in paraplegic and in non paraplegic basketball players
}

\author{
P Calmels MD,${ }^{1} \mathrm{~S}$ Berthouze MD ${ }^{1} \mathrm{~F}$ G Barral MD,${ }^{2} \mathrm{M}$ Domenach MD,${ }^{1} \mathrm{P}$ Minaire \\ $\mathrm{MD},{ }^{1}$
}

${ }^{1}$ Service de Rééducation et Réadaptation Fonctionnelles and GIP 'Exercice' ${ }^{2}$ Service
Central de Radiologie, Hopital Bellevue C.h.u de Saint-Etienne, 42023 France.

Objectives: to study the changes in the strength of the elbow flexors and extensors in paraplegic subjects, and the agonist/antagonist mass and strength ratio in paraplegic wheelchair users.

Method: 10 paraplegic wheelchair basketball players were compared with 10 healthy basketball players. The 20 subjects underwent a clinical and dynamometric isokinetic assessment, and a CT scan measurement of the muscle crosssectional surface area of the flexor and extensor muscles of the elbows.

Results: there was an increase in muscle strength in paraplegic subjects. There was no significant difference in the agonist/antagonist ratio between the dominant and non dominant upper limb in paraplegics whereas such a difference was found in healthy subjects. The muscle mass was increased in the paraplegics, but a correlation between muscle mass and strength was only found in the healthy subjects.

Key words: upper limb muscle strength; paraplegia; basketball players.

\section{Introduction}

The rehabilitation and reintegration of the paraplegic patient in his everyday activities are responsible for a development of the musculature of the upper limbs. Depending on the nature and duration of the physical activities (particularly sports), this development can be more or less prominent. The prime factor at the origin of this source of muscle development is the tendency of the upper limbs to become the propulsive elements for ambulation.

We have carried out a study in paraplegic basketball players of similar sporting level (same level in championship) in an attempt to demonstrate if there is: (1) a difference in muscle strength between the paraplegics and the healthy subjects with a modification in the ratio of the elbow flexor-extensor torque. We have chosen this muscle torque because of its importance in wheelchair propulsion. Grimby has already reported this change of strength in the deltoids but without evaluation of the muscle mass. ${ }^{1} \mathrm{We}$ therefore wanted to study the difference in the muscle mass by evaluating the cross- sectional area of elbow flexors and extensors obtained by computed tomography (CT). The significance of this measurement has been demonstrated by many authors; $;^{2-6}$ (2) a change in the agonist/antagonist and dominant/non-dominant limb ratios in paraplegics compared to healthy subjects, indicating a symmetrization of the upper limbs by analogy with a symmetry of the lower limbs in the healthy subjects. As the upper limbs become the limbs of ambulation they may present changes in the agonist-antagonist ratio similar to those noted after an intensive specific activity ${ }^{7} ;(3)$ a correlation between muscle strength and muscle mass as evaluated by computed tomography. This has already been demonstrated in different populations by several studies. ${ }^{8-13}$

\section{Methods}

\section{Subjects}

The subjects consisted of 20 male basketball players who where divided into 2 subgroups of 10 healthy and 10 paraplegic subjects with a similar level in championship. In the 
paraplegic group the mean age was $31.8 \pm$ 2.3 years, the duration of wheelchair basketball practice was $9.4 \pm 2.5$ years while that of paraplegia was $12.5 \pm 2.4$ years. In the healthy group, the mean age was $28.2 \pm 2.4$ years and the duration of basketball practice was $17.7 \pm 2.4$ years.

\section{Clinical evaluation}

All the subjects underwent a clinical examination that consisted of testing muscles of the upper limb according to Kendall's technique $^{14}$ with a score from 0 to 5 . All the subjects had a score of 5 . There was no evidence of any disease of the upper limbs. For the paraplegics, the highest motor lesional level was T5 and the lowest was L4 (one T5, 4 T6, 2 T10, 2 T12 and one L4). All were permanent wheelchair users.

\section{Dynamometric evaluation}

For each subject, a dynamometric evaluation was performed to estimate the isokinetic muscle strength of elbow flexors and extensors in both upper limbs, using a CYBEX II (Lumex Inc, Ronkonkoma, NY, USA) dynamometer with a pen recorder. The subject was placed in the supine position on the mobile table; for the side tested, the shoulder was placed in abduction at $45^{\circ}$, the elbow was supported by a lateral cushion not limiting extension, the articular axis of the elbow flexion-extension movement was an extension of the dynamometer rotatory axis. The lower limbs rested on a metal support with the hips and knees flexed. To avoid slipping and muscle compensations, the shoulder opposite to the side tested, the trunk, pelvis and lower limbs were stabilised on the examination table by self gripping strips. On the same non tested side, the subject had a stabilising lateral handle at his disposal. The level arm of the dynamometer (mobile during the flexion-extension movement) presented a handle held by the patient in the intermediate position of pronation-supination of the wrist and adjustable according to the length of the forearm to permit total flexion-extension of the elbow.

After teaching each subject how to use the dynamometer, the value of the elbow flexor-extensor torque was evaluated, starting with the dominant side. The tests were performed at different angular velocities: $30,60,120,150^{\circ}$. For each test and from the initial position of extension, the patient made 3 complete amplitudes of flexionextension. A rest period of 60 seconds was observed between each test.

For each subject, we also determined (1) the maximal value of the concentric peak torque for elbow flexor and extensor muscles in $\mathrm{N} / \mathrm{m}$ after 3 complete ranges of motion and for each angular velocity; and (2) the flexor and extensor impulsive strength (IMP), the value of the torque at two tenths of a second from the beginning of one movement of flexion and one movement of extension at an angular velocity of $60^{\circ}$ with a damping setting of 2 to control overshoot in the beginning of the range of motion.

\section{CT evaluation}

On another day, the cross-sectional area of the flexor and extensor elbow was obtained on a sagittal section of the arm using an ELSCINT (R) EXEL 1800 computer tomography scanner. The section was obtained at $10 \mathrm{~cm}$ from the olecranon with the patient in the prone position, the shoulder completely flexed and the elbow in extension for simultaneous examination of the 2 arms. The outlines of the muscle groups (flexors: biceps brachii and brachialis; extensors: triceps brachii) were manually determined according to tissue density and the crosssectional area was measured by the computer. All areas were calculated by the same investigator.

\section{Statistical analysis}

The Student parametric t-test was used to determine the statistical significance of the differences between the means in each group and we considered a difference to be significant for a $p$ value $<0.05$. Spearman's correlation coefficient was used to establish the correlation between cross-sectional area and torque and the correlation coefficients were considered to be significant for $p$ values $<0.05$. 


\section{Results}

The paraplegics' muscle strength was superior to that of healthy subjects for flexors and extensors, when the dominant and non dominant limbs were compared. This difference was significant for angular velocities greater than $60^{\circ}(p<0.05)$ and for the impulsive strength (IMP) $(p<0.05)$ of elbow flexors (Fig 1a) and extensors (Fig 1b).

The elbow extensor-flexor torque ratio was equal to or greater than 1 except for angular velocity of $150^{\circ}$ in the paraplegics' dominant limb. In healthy subjects, the difference between the ratio in the dominant and the non dominant limb was significant $(p<0.05)$ for angular velocities of $60^{\circ}$, $120^{\circ}$ and $150^{\circ}$ and for impulsive strength, but was not significant in paraplegics (Table I).

The cross-sectional area of elbow flexors and extensors was significantly greater in paraplegics compared to healthy subjects for the dominant and the non dominant limb $(p<0.05)$ (Table II).

The strength/cross-sectional area quotient was used to establish an index of $\mathrm{Nm} / \mathrm{cm} 2$ which was superior in healthy subjects to that in paraplegics. However, this difference was not significant.

There was a significant correlation $(p<0.05)$ between muscle cross-sectional area (evaluated by $\mathrm{CT}$ sections) and the maximal torque product by extensors and flexors for the whole population (healthy and paraplegic subjects) on the dominant and non dominant sides. However, the results were different in the 2 groups when the correlation was established separately for healthy $(p<0.05)$ and for paraplegic subjects $(p>0.05)$ for flexors and for extensors.

\section{Discussion}

Our results show that for impulsive strength and at high but not low velocity of movement, paraplegics have a significantly greater muscle strength of the elbow flexors and extensors than do healthy subjects, and the muscle cross-sectional area of the upper limbs is significantly greater in paraplegics than in healthy subjects.
The elbow extensor-flexor ratio of the dominant and non dominant limbs was comparable in the 2 populations, but the difference between the 2 sides was significant in the healthy subjects only and not in the paraplegics. This may suggest an attenuation of the asymmetry in dominant/nondominant limbs in paraplegics.

Although there was a significant correlation between muscle strength and crosssectional area in healthy subjects, this correlation did not exist in paraplegics, and the muscle strength/cross-sectional area quotient was lower in paraplegics, but not significantly so.

It should be noted that the torques produced by elbow flexors and extensors in healthy subjects for angular velocities of $30^{\circ}$ (values ranging from 30 to $50 \mathrm{~N} / \mathrm{m}$ ) and our values for muscle cross-sectional area obtained by $\mathrm{CT}$ evaluation on the dominant side were similar to those obtained by Schantz. ${ }^{13}$

The paraplegics were stronger than the healthy subjects in our tests but this difference was only significant for impulsive strength and for strength at high velocity. These high velocities are close to those used for wheelchair propulsion. A similar development to that seen in the strength of basketball players has already been demonstrated by Grimby ${ }^{1}$ who showed that the strength of the upper limb muscles was considerably greater in the wheelchair players; according to this author, the strength of the deltoid muscle can be at least $50 \%$ greater in an active paraplegic than in a moderately trained healthy subject.

However, our results differed by the fact that in his study ${ }^{1}$ of the deltoid muscles the strength in paraplegics was superior to that in controls especially at low angular velocity. The differences lessened at high angular velocity, in contrast to the results of our study. According to Grimby, the high proportion of slow fibres found in paraplegics explains the difference in the strength of the deltoid muscle. However, if we refer to the study by Thorstenson ${ }^{15}$ in which the movements were more rapid, the rapid type II and even II muscle fibres may be involved, suggesting that paraplegics' elbow flexors and extensors would be richer in type II 

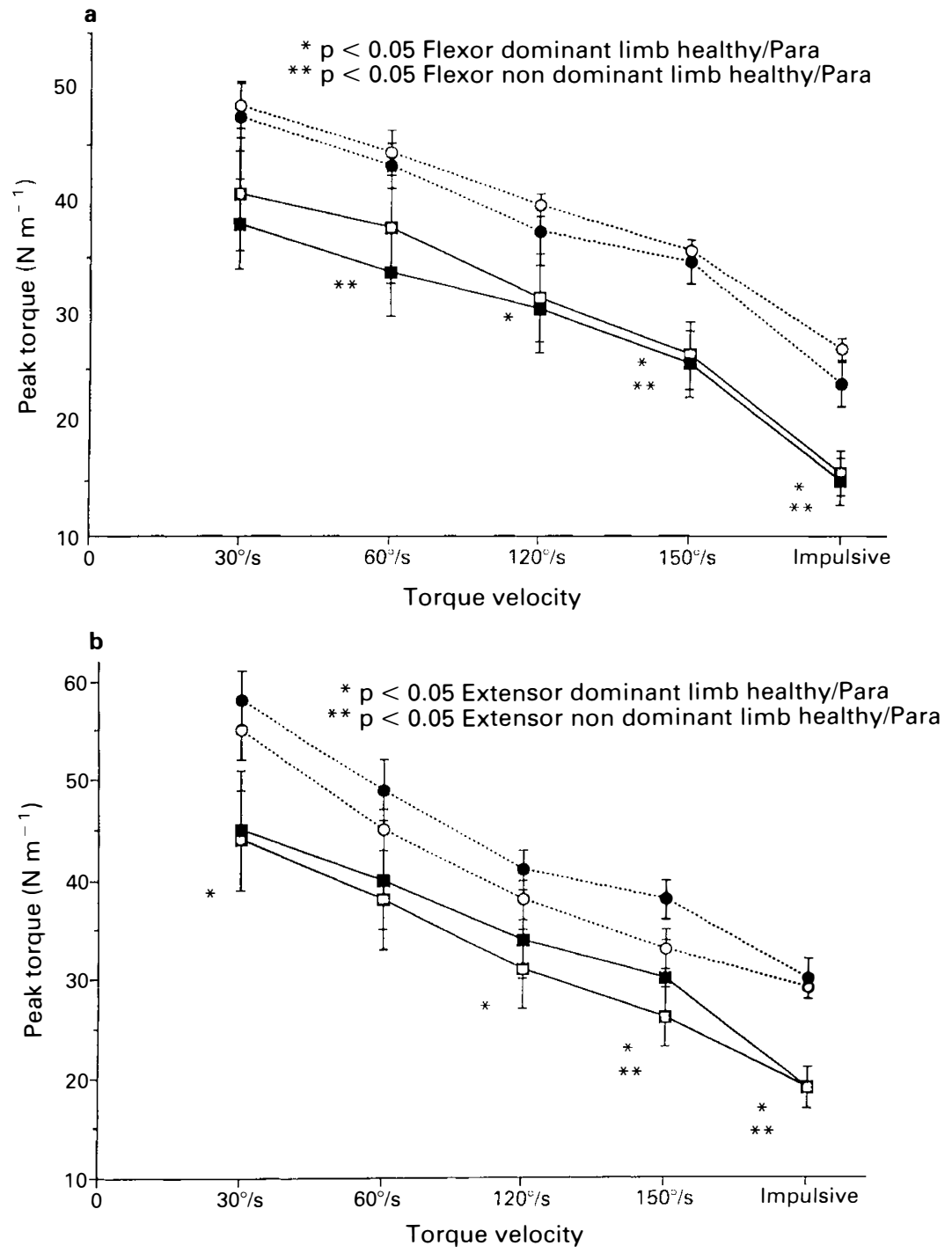

Figure 1a Mean peak torque for elbow flexors. Dominant $\square$ and non dominant $\boldsymbol{\square}$ limb healthy subjects.

Dominant $\odot$ and non dominant $\bullet$ limb paraplegic subjects.

The paraplegics' muscle peak torque is superior to that of healthy subjects for the dominant and non dominant limbs. This difference is significant $(p<0.05)$ for angular velocities $120^{\circ}, 150^{\circ}$ and impulsive movement in the case of dominant limbs, and for $60^{\circ}, 150^{\circ}$ and impulsive movement for the non dominant limbs.
Figure 1b Mean peak torque for elbow extensors.

Dominant $\square$ and non dominant $\square$ limb healthy subjects.

Dominant $\bigcirc$ and non dominant $\bullet$ limb paraplegic subjects.

Paraplegic's muscle peak torque is superior to that of healthy subjects for the dominant and non dominant limbs. This difference is significant $(p<0.05)$ for angular velocities $30^{\circ}, 120^{\circ}$, $150^{\circ}$ and impulsive movement in the case of dominant limbs, and for $150^{\circ}$ and impulsive movement for the non dominant limbs. 
Table I Mean values of the extensors/flexors ratio of the elbow

\begin{tabular}{lcccccc}
\hline EF & $\begin{array}{c}\text { Dominant limb } \\
\text { healthy subjects }\end{array}$ & $\begin{array}{c}\text { Non dominant } \\
\text { limb healthy } \\
\text { subjects }\end{array}$ & $\begin{array}{c}\text { Dominant limb } \\
\text { paraplegic subjects }\end{array}$ & $\begin{array}{c}\text { Non dominant limb } \\
\text { paraplegic subjects }\end{array}$ \\
\hline $30^{\circ}$ & $1.1 \pm 0.2$ & $\mathrm{NS}$ & $1.2 \pm 0.8$ & $1.1 \pm 0.2$ & $\mathrm{NS}$ & $1.2 \pm 0.5$ \\
$60^{\circ}$ & $1.0 \pm 0.1$ & $\mathrm{~S}$ & $1.2 \pm 0.8$ & $1.0 \pm 0.1$ & $\mathrm{NS}$ & $1.1 \pm 0.5$ \\
$120^{\circ}$ & $1.0 \pm 0.1$ & $\mathrm{~S}$ & $1.1 \pm 0.4$ & $1.0 \pm 0.1$ & $\mathrm{NS}$ & $1.1 \pm 0.3$ \\
$150^{\circ}$ & $1.0 \pm 0.1$ & $\mathrm{~S}$ & $1.2 \pm 0.5$ & $0.9 \pm 0.1$ & $\mathrm{NS}$ & $1.1 \pm 0.1$ \\
Impulsive & $1.3 \pm 0$ & $\mathrm{~S}$ & $1.1 \pm 0.1$ & $1.1 \pm 0$ & $\mathrm{NS}$ & $1.3 \pm 0.1$ \\
strength & & & & & & \\
\hline
\end{tabular}

Table II Comparison between the mean cross-sectional area in $\mathrm{mm}^{2}$ of muscle in healthy and paraplegic subjects

\begin{tabular}{lcccc}
\hline Mean & Healthy & Paraplegic & T-tests & $p$ \\
\hline Flexors dominant limb & 2382.0 & 3166.9 & 3.35 & $<0.05$ \\
& \pm 124.86 & \pm 142.13 & & \\
Extensors dominant limb & 1679.7 & 2484.0 & 4.07 & $<0.05$ \\
& \pm 114.39 & \pm 144.7 & & $<0.01$ \\
Flexors non dominant limb & 2415.7 & 3164.0 & 3.11 & $<0.05$ \\
& \pm 11.84 & \pm 167.8 & & \\
Extensors non dominant limb & 1618.1 & 2572.5 & 4.19 & \\
& \pm 96.06 & \pm 188.06 & & \\
\hline
\end{tabular}

fibres. Moreover, it is known that training favours the development of type II fibres. ${ }^{16-18}$ Although in our sample each subject did not follow a specific and similar training but simply undertook intensive sporting and physical activity, differential fibre development may have occurred. However, the specificity of type II fibres to develop greater tension/mass at high velocity training is much disputed in various studies $^{9,12,13,15}$ and it would be advisable to perform muscle biopsies to confirm it.

On the other hand, the differences in relation to the results of the other study ${ }^{1}$ more particularly concerned specific muscle activity; in paraplegics, the deltoid muscles (used for transfer) have a more static activity, while the elbow flexors and extensors (with a role in wheelchair propulsion) have a more dynamic activity. A biomechanical analysis of wheelchair propulsion might provide a better assessment of the activity of these muscles and the movement velocities, but it appears that variations in the mode of wheelchair propulsion according to the lesional level and trunk musculature may exist. ${ }^{19}$

Paraplegic subjects present an extensorflexor ratio for the dominant and non dominant limbs similar to that of healthy subjects, but, in paraplegic subjects only, there is no statistically significant difference between the 2 sides. This difference may be due to the specific activity of the sports training as we have previously shown in a study of tennis players. ${ }^{7}$ We found a difference in the extensors/flexors ratio of the wrist for healthy and untrained subjects between the dominant and non dominant sides. This difference was only significant for high velocity $\left(120^{\circ}\right)$ but not for low velocity $\left(30^{\circ}\right)$ and isometric torque.

If, as proposed by various studies for the lower limbs ${ }^{20-23}$, we accept that agonist/ antagonist ratio is a functional and individual index of the balance between the 2 
opposite muscle groups at different speeds of movement, our results seem to demonstrate a tendency towards functional symmetry in paraplegic upper limbs. The extensor/flexor ratio of the elbow was not statistically different on the opposite side, as in the lower limbs, and particularly at high velocity, which is necessary for wheelchair propulsion. However, for the measurement of the muscle strength only, it seems that a dominant side always persists. Further studies concerning the difference in the agonist/antagonist ratio in healthy and untrained subjects are required.

Finally, in paraplegic subjects the crosssectional area evaluated by CT studies was greater than that in healthy subjects. In view of the exercise they perform, this reflects muscle hypertrophy of elbow flexors and extensors. ${ }^{24}$ However, whereas many studies found a significant correlation between muscle strength and cross-sectional area evaluated by CT, regardless of age, ${ }^{25}$ sex,${ }^{6}$ and activity, ${ }^{9,11}$ we obtained discordant results: in our study, this correlation was only significant when the whole population (paraplegic + healthy) or the healthy group was considered. In paraplegics, we did not find this correlation because muscle strength was less than that predicted from the crosssectional area. Indeed, the strength/crosssectional area quotient was less than that in healthy subjects.

This difference may be due to different factors: histological, neurological or statistical. In our study, strength was greater for paraplegic than for healthy players for high angular velocity. Many studies admit that, at high angular velocities, type II muscle fibres present an ability to develop a muscle strength superior to that of type I fibres, ${ }^{6.9 .26}$ but we cannot be sure that the difference was only due to the changes in histological structure. Although the study of Grimby ${ }^{1}$ showed hypertrophy of the 2 types of fibres (I and II) in the deltoid muscles of paraplegic subjects, we cannot compare the results because the activity of the elbow flexors and extensors is different for wheelchair propulsion.

Several studies of electromyographic activity and muscle strength show a relation between the increase in electromyographic activity and strength. This relationship follows a double exponential, ${ }^{27-29}$ one of which would correspond to fibre contractile capacities, the other to motor unit recruitment, but changes related to modifications in excitability, learning, and motor recruitment according to the muscles and movement velocity ${ }^{30}$ cannot be ruled out.

In the case of our sample, the variation in the strength/cross-sectional area relationship can be due to neurological effects. Firstly, there is a difference of training for each group and each subject, because the paraplegics had different durations from the injury and different programmes of rehabilitation. Each group had different functional training for playing basketball. Several studies indicate the interaction of different factors in the hypertrophy and neural factors in the development of strength, ${ }^{31-34}$ and some authors think that this difference is due to the fact that morphological and neurological factors do not follow a similar time course during training. Secondly, as each limb was tested independently, the principle of 'defect in bilateral strength' reported by Enoka $^{30}$ can partially explain the decrease in the strength of paraplegics relative to cross-sectional area, as their daily training induced a maximal and concurrent activation of the contralateral limb and the 2 homologous muscles for ambulation. This effect would be secondary to a decrease in slow fibre activity on electromyography. To test this theory it will be necessary to compare this effect by electromyography in paraplegic and healthy subjects.

Statistically, the difference in the correlation between peak torque and crosssectional area between paraplegic and healthy basketball players may be due to the small and inhomogeneous sample. This could explain the absence of a significant difference in the strength/cross-sectional area quotient between paraplegic and healthy subjects.

\section{Conclusion}

Our study describes the development of muscle strength and mass in paraplegic basketball players and the difference in the 
muscle peak torque and cross-sectional area in relation to healthy basketball players. It suggests the importance of a neuromuscular factor. It would appear necessary to study the course of the histological structure and electromyographic activity of the upper limbs in paraplegic subjects during their rehabilitation programme in order to propose an adapted training programme for sports as well as for everyday life activities.

\section{References}

1 Grimby G, Broberg C, Krotkiewska I, Schoonens J (1980) Aerobic capacity, muscle strength and fiber composition in young paraplegics. First International Medical Congress on Sports for the Disabled, Ustaoset, Norway, February 1-8: 13-17.

2 Brenton DP, Edwards RHT, Grindrod SR, Tofts PS (1981) Computerized X-ray tomography to determine human skeletal muscle size and composition in health and disease. J Physiol 317: 3.

3 Bulcke JA, Crolla D, Termotte, JL, Baert A, Palmers Y, Van der Bergh R (1981) Computed tomography of muscle. Muscle Nerve 4: 67-72.

4 Bulcke JA, Termotte JL, Palmers Y, Crolla D (1979) Computed tomography of the human skeletal muscular system. Neuroradiology 17: 127-136.

5 Grindrod S, Tofts P, Edwards R (1983) Investigation of human skeletal muscle structure and composition by X-ray computerised tomography. Eur J Clin Invest 13: 465-468.

6 Maugham RJ, Watson JS, Weir J (1983) Strength and cross-sectional area of human skeletal muscle. J Physiol 338: $37-49$.

7 Calmels P, Salfati-Rieffel C, Abeillon G, Domenach M, Minaire P (1988) Etude dynamométrique de la flexion/extension du poignet chez le joueur de tennis: approche étiopathogénique de l'épicondylite. Ann Réadapt Méd Phys 31: 482-491.

8 Chapman JP, Grindrod SR, Jones DA (1984) Cross-sectional area and force production of the quadriceps muscle. J Physiol 353: 53 .

9 Johansson C, Lorentzon R, Sjostrom M, Fagerlund M, Fugl-Meyer AR (1987) Sprinters and marathon runners. Does isokinetic knee extensor performance reflect muscle size and structure? Acta Physiol Scand 130: $663-669$.

10 Kristensen JH, Hansen TI, Saltim B (1980) Cross-sectional and fiber size changes in the quadriceps muscle of man with immobilization and physical training. Muscle Nerve May/June: 275-276.

11 Maugham RJ, Watson JS Weir J (1983) Relationships between muscle strength and muscle cross-sectional area in male sprinter and endurance runners. Eur J Appl Physiol 50: 309-318.

12 Nygaard E, Houston M, Suzuki Y, Jorgensen K, Saltin B (1983) Morphology of the brachial biceps muscle and elbow flexion in man. Acta Physio! Scand 117: 287-292.

13 Schantz P, Randall-Fox E, Hutchison W, Tyden A, Astrand PO (1983) Muscle fibre type distribution, muscle cross-sectional area and maximal voluntary strength in humans. Acta Physiol Scand 117: 219-226.

14 Kendall HO, Kendall FP, Wadsworth GE (1971) Muscles: Testing and Function. Williams \& Wilkins, Baltimore: 284 .

15 Thorstensson A, Grimby G, Karlsson J (1976) Force-velocity relations and fiber composition in human knee extensor muscles. J Appl Physiol 40: 12-16.

16 Astrand PO (1987) Exercise physiology and its roles in disease prevention and in rehabilitation. Arch Phys Med Rehabil 68: 305-309.

17 Hermansen L, Vollestad NK, Staff PH, Daljord OD, Gronnerod O (1983) The effect of immobilization and training on strength and composition of human skeletal muscle. In: Space Physiology. Cepadues, CNES Toulouse: $255-266$.

18 Saint-Pierre D, Gardiner PF (1987) The effect of immobilization and exercise on muscle function: a review. Physiother Can 39: 24-29.

19 Steadward R (1980) Analysis of the wheelchair sport events. First International Medical Congress on Sports for the Disabled, Ustaoset Norway, February 1-8: 184-192.

20 Agre JC, Casal DC, Leon AS, Mc Nally MC, Baxter TL, Serfass RC (1988) Professional ice hockey players: physiologic, anthropometric, and musculoskeletal characteristics. Arch Phys Med Rehabil 69: 188-192.

21 Alexander MJL (1990) Peak torque values for antagonist muscle groups and concentric and excentric contraction types for elite sprinters. Arch Phys Med Rehabil 71: 334-339.

22 Kannus P, Jarvinen M (1990) Knee flexor/extensor strength ratio in follow-up of acute knee distorsion injuries. Arch Phys Med Rehabil 71: 38-41.

23 Macintyre D, Wessel J (1988) Knee muscle torque in pattelofemoral pain syndrome. Physiother Can 40 (1): 20-23.

24 Fox ED, Mathews DK (1984) Bases Physiologiques de L'activité Physique. Vigot, Paris: 404.

25 Young A, Stokes M, Round JM, Edwards RHT (1983) The effect of high-resistance training on the strength and cross-sectional area of the human quadriceps. Eur J Clin Invest 13: 411-417. 
26 Young A (1984) The relative isometric strength of type I and type II muscle fibres in the human quadriceps. Clin Physiol 4: 23-32.

27 Metral S, Cassar G (1981) Relation between force and integreted EMG activity during voluntary isometric anisotonic contraction. Eur J Appl Physiol 46: 185-198.

28 Soderberg GL, Cook M (1984) Electromyography in biomechanics. Phys Ther 64 (12): 1813-1820.

29 Solomonow M, Baratta R, Zhou BH, Shoji H, D'Ambrosia R (1986) Historical update and new developments on the EMG-force-relationships of skeletal muscles. Orthopedics 9 (11): 1541-1543.

30 Enoka RM (1988) Muscle strength and its development: new perspectives. Sports Med 6: $146-166$.

31 Cote C, Simoneau JA, Lagasse P, et al (1988) Isokinetic strength training protocols: do they induce skeletal muscle hypertrophy? Arch Phys Med Rehabil 69: 281-285.

32 Petersen S, Wessel J, Bagnall K, Wilkins H, Quinney A, Wenger H (1990) Influence of concentric resistance training on concentric and excentric strength. Arch Phys Med Rehabil 71: 101-105.

33 Komi PV (1986) How important is neural drive for strength and power development in human skeletal muscle? Int Series Sport Sci 16: 515-529.

34 Grimby G (1985) Progressive resistance exercise for injury rehabilitation. Special emphasis on isokinetic training. Sports Med 2: 309-315. 